\title{
A Game-Theoretic Analysis to Resolve the Tussle in Cloud Storage Services
}

\author{
Libin Yang and Xiaoyan Cai* \\ Department of Information Engineering, Northwest Agricultural and Forestry University, Yangling, Shaanxi, China
}

Received: 24 Jun. 2013, Revised: 29 Oct. 2013, Accepted: 30 Oct. 2013

Published online: 1 May. 2014

\begin{abstract}
Cloud computing has not only gained enormous popularity, but also been deployed successfully in large scale realworld applications. However, the popularity of the cloud storage also leads to the dilemma between end-user(EU) and cloud storage provider(CSP), i.e, the EU focuses its attention on acquiring cheap cloud storage services, while CSP concerns how to maximize its own revenue by setting proper price. The noncooperative decisions of EU and CSP will possibly result in corresponding loss welfare of the both parties. In the case of the cloud storage business, we model the interactions between EU and CSP as Stackelberg game and analyze the equilibrium strategies of the two parties. We show that the welfare of the two parties involved in the cloud storage business can be further enhanced to achieve the system optimality by applying Nash Bargain Solution(NBS). Our theoretical analysis and empirical studies both show that the bargain solution can result in a win-win outcome.
\end{abstract}

Keywords: Cloud Storage, Pricing, Game Theory, Pareto Improvement

\section{Introduction}

As a hot service delivery platform in the field of service computing [1], cloud computing has not only attracted substantial research attention, but also been deployed successfully in large scale real-world applications. Cloud computing is operated on centralized facilities deployed by the third-party. It provides convenient computing and storage services so that individuals and organizations are able to outsource their IT requirements to remote data centers, paying for only what they use. As a novel business of infrastructure, cloud computing is growing rapidly.

Cloud often provide different services, among which storage cloud service is the most popular one. Several service vendors currently provide storage cloud service online. These storage cloud service allows anyone purchase storage capacity online, by paying a certain fee for the storage they use. For example, Amazon.com offers data storage priced by the gigabyte-month. The interested users, e.g., research institutions, could easily purchase this kind of cloud-based services with QoS guarantee by signing a cloud storage contract with service provider. The contract sets out the division of responsibility for both parties, i.e., an end user and a cloud service provider, and also specifics the payment scheme of cloud service. The cloud vendors often employ usage-based pricing, in which users pay proportionally to the amount of time and the amount of resources they use. Comparing with the other more sophisticated pricing models, the usage-based payment, which has been widely used in real world, is more popular since it is simple and efficient.

For a storage cloud provider, its profits depend on selling cloud storage. One important goal of cloud provider is how to carefully set service price so as to maximize the corresponding revenue. Meanwhile, with the significant growth of society's storage requirements and the availability of pay per-use online storage, EUs also have their concerns, i.e., how to maximize its own utility when a EU considers the using of storage clouds in face of the usage price given by the cloud provider. However, the revenue maximization decisions of the two parties, i.e., CSP and EUs, is often a critical dilemma. It will result in corresponding welfare loss of both sides since both parties are rational and make decisions in order to only maximize their own revenues non-cooperatively. There are serval literatures $[2,3,4]$ describing theoretical pricing models to assist cloud providers in enhancing their revenues decisions. Comparatively, very little attention has been focused on handling cloud storage in

\footnotetext{
*Corresponding author e-mail: xiaoyanc@nwsuaf.edu.cn
} 
the form of game theory. The game theory is capable of measuring the efficiency of both parties where the noncooperative factors between the two parties are also taken into consideration.

The goal of this paper is to shed some lights on the deployment and evolution of cloud storage business. We hope that our analysis could inspire the decision makers of EUs to acquire cheaper compute resources, where they may form a coalition that is capable of bargaining with cloud provider. On the other hand, the results of the analysis is able to develop qualitative pricing guidelines for successful cloud service deployments and enhance the market share of the cloud storage providers.

Specially, in the case of the cloud storage business, we model the interactions between EU and CSP as Stackelberg game and analyze the equilibrium strategies of the two parties. We show that the welfare of two parties involved in the cloud business can be further enhanced to achieve the system optimality by applying Nash Bargain Solution(NBS). Our theoretical analysis and empirical studies both show that the game existing in cloud storage service can result in a win-win outcome.

The rest of the paper is organized as follows. In section 2, we review some related work. We formulate the interactions between EU and CSP as a noncooperative game and get the equilibrium strategies of the two parties in Section 3. In Section 4, we investigate the pareto improvement by applying NBS. We evaluate the theoretical analysis In Section 5. The conclusion is presented in Section 6.

\section{Related Work}

Cloud computing becomes more and more popular recently. Cloud computing provides access to large amounts of data and computational resources that can be acquired on-demand. Cloud often provides three different services: hardware, software and storage. For example, Amazon provides a cloud storage service namely EC2(Elastic Cloud Computing) Service [5], where storage infrastructures are open to public access with a resource usage-based pricing model. Another open source cloud implementation is Eucalyptus [6], which provides compatible interface to Amazons EC2. Eucalyptus allows people to set up a cloud infrastructure and thus let them get prior to buying commercial services.

The cloud service often involves many management methods and price models since service providers always seek to maximize their revenue. Various resource management algorithms were published in some papers [7] [2] [3]. These management algorithms concentrate on task scheduling and require communications among tasks. On the contrary, current cloud users mainly focus on price or on the nature quality of cloud service.

Over the past few years, combining economic models with game theoretic analysis has become increasingly popular in network economics. Park et al. [4] constructed a formal game theoretic model to investigate the issues of incentives in file sharing. Antoniadis et al. [8] developed a theoretical framework that abstracts the shared contents as public goods and a social planner that improves the cooperation through a proper pricing scheme. Game theory also can be applied to study the problems existing in grid or cloud service from the aspect of resource owners [9] [10] [11]. However, there is still lack of practicable solution for cloud computing systems since the tussle existing in the business of cloud service is not only a technical problem at the network aspect, but also an economic problem at the business aspect. Therefore, for the first step, this paper presents a game-theoretic analysis to resolve the dilemma of cloud service from business aspect.

\section{Basic Cloud Storage Contract}

In this section, we study the economic relationship between an EU and a CSP existing in cloud storage business.

\subsection{Economic Model for Cloud Storage Business}

Consider a cloud storage scenario with an EU and a CSP. EU is interested in cloud storage service provided by CSP. Normally, the CSP sets price for the EU by providing cloud storage services with QoS guarantee, which is bounded by a cloud storage contract. According to the contract, the EU pays for the cloud storage services, and in return it will be served with a contractual storage space by CSP. The contract mainly consists of three components: 1) the performance component which specifies the agreed storage space metric, i.e., the storage space that CSP should provide; 2) the payment component which specifies the payment of EU; 3) the time component which defines the contract's life time, i.e., the period the storage lasts.

Without loss of generality, we assume CSP adopts the linear tariff in this cloud storage business, i.e., CSP sets constant price per-unit storage space for providing cloud storage service. The EU determines its storage request by taking account of its price sensitivity. Indeed, the CSP also has to undertake amount of cost for providing such services, e.g, the operating cost. We make the following assumption on the cost function of the CSP:

Assumption 1The cost of the CSP is a function of the storage resource $B$ requested by the $E U$, denoted as $C_{C S P}(B)$, which is differentiable and strictly convex. The first-order derivative of $C_{C S P}(B)$ is denoted by $C_{C S P}^{\prime}$. 
Thus, CSP's revenue function can be expressed as:

$$
R_{C S P}=P \cdot B-C_{C S P}(B)
$$

where $P$ is the price of per-unit cloud storage space, $B$ is the storage space that CSP provides to meet the EU's requirement. $P \cdot B$ is the payment of $\mathrm{EU}$.

The EU pays for the services and derives its utility from high quality and secure cloud storage experience. We represent EU's revenue function as:

$$
R_{E U}=U(B)-P \cdot B
$$

where $U(B)$ indicates the utility that the EU derives from the given storage. Due to the marginal effect [12], we have the following assumption:

Assumption 2The experience of EU on the feasible consumption of cloud storage resouce $B$ can be represented by a continuous utility function $U(B)$, which is strictly increasing and concave. Formally, the first-order derivative of $U(B)$ is denoted by $U^{\prime}(B)$.

\subsection{Equilibrium in Basic Cloud Storage Contract}

Given the revenue function of EU and CSP, we now investigate their strategic interaction when they selfishly maximize their own revenue. In the cloud storage business, CSP has the power to determine the price of per-unit cloud storage resource. In what follows, EU chooses its storage resource request to optimize its own revenue in response to the price given by the CSP. It is naturally a two-stage of Stackelberg game, where in the first stage CSP acts as a leader by setting price $P$, and in the second stage the EU acts as a follower to determine its best storage resource consumption $B$ in face of the price $P$, which can be represented as a storage resource demand function $B=D(P)$. They seek to maximize their own revenues by determining the strategies of price $P$ and storage resource request $B$, respectively. Assume CSP knows the storage resource demand function of EU, so that CSP can choose its optimal price to maximize its revenue by expecting EU's best response. We derive the sub-game perfect equilibrium for the cloud storage business by applying the concept of backward induction, which works as follows:

Firstly, in the second stage, for a given price of per-unit storage resource $P$, EU determines its optimal storage resource demand $B^{*}=D(P)$ along with their corresponding revenues, i.e., EU takes price $P$ given by CSP as input and decides optimal storage resource consumption $B^{*}=D(P)$ as outcome. Then back in the first stage, CSP, acting as the leader of the Stackelberg game, is aware of the storage resource requirement of EU. Because $B^{*}=D(P)$ has became common knowledge as the game is played since CSP has obtained demand function of the EU, CSP can choose its optimal price $P^{*}$ to maximize its revenue by taking consideration of the EU's storage resource demand function $B^{*}=D(P)$. Hence, the subgame perfect equilibrium $P^{*}, B^{*}$ of the full game can be found. The detailed analysis is listed in Theorem 1.

Theorem 1Given Assumptions 1 and 2 hold, there exists a Nash equilibrium for the cloud storage contract and the equilibrium strategy profile $\left(P^{*}, B^{*}\right)$ satisfies:

$$
-B^{*} / D^{\prime}\left(P^{*}\right)=P^{*}-C_{C S P}^{\prime}\left(B^{*}\right)
$$

where $B^{*}=D\left(P^{*}\right)=U^{\prime-1}\left(P^{*}\right), \quad B=U^{\prime-1}(P)$ is the inverse function of $P=U^{\prime}(B)$.

Proof.First, for a given price of per-unit storage $P$, EU aims to determine its optimal storage resource demand by solving the following problem

$$
\max _{B \geq 0} R_{E U}=U(B)-P \cdot B
$$

By applying the first order optimality condition with respect to $B$, we can obtain the EU's best response $P=U^{\prime}(B)$, where the price of per-unit storage $P$ equals to marginal utility $U^{\prime}(B)$. We can characterize the EU's best response of price $P$ to a storage demand function $B^{*}=D(P)=U^{-1}(P)$. CSP simply uses its revenue to determine its strategy, i.e., setting a proper price. Thus, by expecting EU's response $B=D(P)$, CSP aims to solve the following revenue maximizing problem:

$$
\max _{P \geq 0, B \geq 0} R_{C S P}(P, B)
$$

By applying the first order condition with respect to $P$, we obtain $\frac{\partial R_{C S P}}{\partial P}+\frac{\partial R_{C S P}}{\partial B} D^{\prime}(P)=0$. Rearranging it, yields

$$
-\frac{\partial R_{C S P}}{\partial P} / \frac{\partial R_{C S P}}{\partial B}=D^{\prime}(P)
$$

where $\frac{\partial R_{C S P}}{\partial P}$ and $\frac{\partial R_{C S P}}{\partial B}$ are the changing rates of CSP's revenue with the variables $P$ and $B$, respectively. Recall that $R_{C S P}=P \cdot B-C_{C S P}(B)$ indicated in Eq. 1, Eq. 6 can be further reduced to Eq. 3, where the optimal price $P^{*}$ can be found. Meanwhile the final optimal storage resource requirement is also determined by $B^{*}=D\left(P^{*}\right)=U^{\prime}\left(P^{*}\right)$. The strategy profile $\left(P^{*}, B^{*}\right)$ is the subgame perfect Equilibrium that represents the steady state of the Stackelberg game.

Notice that the left side of Eq. 6 can be interpreted as the marginal rate of substitution of CSP's revenue, indicated in Figure.1(a) as the slope of CSP's indifferent-revenue curve $R_{C S P}$. The right side of Eq. 6 is the marginal storage resource demand of EU. We can employ a geometry based argument to explain how to get the equilibrium strategy profile $\left(P^{*}, B^{*}\right)$. Since CSP will seek to maximize its revenue by taking consideration of 
EU's demand function $D(P)$, we can first plot the curve of EU's storage resource demand function $B=D(P)$. In what follows, CSP will choose a point of curve $B=D(P)$ that makes its revenue maximized. From Figure.1 (a), we can see that the upper the CSP's indifferent revenue curve is, the larger CSP's revenue will be. Therefore, CSP will achieve the highest feasible revenue by choosing the optimal price $P^{*}$ such that the indifferent revenue curve is tangent to the demand function curve $D(P)$, depicted in Figure.1(a) as the point $Q$. Meanwhile, the equilibrium storage resource consumption of EU can be obtained according to the demand function $B^{*}=D\left(P^{*}\right)$. The equilibrium point $Q=\left(P^{*}, B^{*}\right)$, in essence, is the point that the indifferent-revenue curve $R_{C S P}$ and $R_{E U}$ intersect.

Note that marginal utility function $U^{\prime}(B)$ diminishes with $P$ increases according to the marginal effect [12]. It implies that the demand function $D(P)$ is also decreasing with $P$ increasing. $B=D(P)$ reflects the rational response of EU to the price, which is well consistent with the common sense. We also observe that the left side of Eq. 3 is CSP's marginal revenues, which equals to price of per-unit storage resource minus marginal cost. The right side of Eq. 3 can be interpreted as the demand elasticity which represents EU's sensitivity to the changing price, it also implies that the storage resource requirement is relatively inelastic when price is low, but it becomes more elastic when price increases, which is consistent with the nature of internet it services: once the price goes beyond a certain threshold, it becomes a much more important factor influencing EU's consumption. The implication of Eq. 6 shows that the CSP's revenue is determined by EU's demand elasticity. The larger EU's demand elasticity is, the more revenue CSP may obtain.

\section{Pareto Improvement in Basic Cloud Storage Contract}

We have obtained the equilibrium Stackelberg game. However, it is shown that the equilibrium of a game may not achieve full efficiency [13]. A classic concept in game theory quantifying the efficiency of a game is Pareto efficiency. In the context of the cloud storage contract, we find that the strategy profile $\left(P^{*}, B^{*}=D\left(P^{*}\right)\right)$ is the subgame perfect equilibrium, but it is not a equilibrium with Pareto efficiency. The implication of the analysis is that it will result in corresponding social revenue loss as both CSP and EU try to increase their own revenues selfishly by adjusting their own strategies. In fact, if both sides make some compromise, i.e., CSP decreases price and EU buys more storage resource, they may derive a better revenue. Conceptually, we can illustrate this in Figure.1(b). In the shaded areas enclosed by two curves $R_{C S P}$ and $R_{E U}$, the revenues of CSP and EU can be both improved to $\hat{R_{C S P}}$ and $\hat{R_{E U}}$ from $R_{C S P}$ and $R_{E U}$, respectively. It should be noted that for Figure.1(b), the lower curve $\hat{R_{E U}}$ represents the higher revenue for EU, so EU attains a higher revenue by reducing price.
Following the above analysis, our goal is to design an optimal cloud storage contract so that the system consisting of the EU and CSP can achieve Pareto efficiency. Essentially, we notice that the payment $P \cdot B$ serves only to allocate the total revenue between the EU and CSP. We may find an optimal cloud storage contract that improves the revenue of both parties through a bargain process. With this concept, we can use Nash Bargain Solution(NBS) [14] to get a Pareto efficient contract. Mathematically, we seek the optimal strategy profile $\left(P^{*}, B^{*}\right)$ by solving the following problem

$$
\max _{P \geq 0, B \geq 0} R_{E U}(P, B) \cdot R_{C S P}(P, B)
$$

The detailed result is presented in the following theorem.

Theorem 2Given that Assumptions 1 and 2 hold, there exists an optimal Nash equilibrium with pareto efficiency for the cloud storage contract and the equilibrium strategy profile $\left(P^{*}, B^{*}\right)$ satisfies:

$$
P^{*}=U^{\prime}\left(B^{*}\right)=C_{C S P}^{\prime}\left(B^{*}\right)
$$

Proof. The optimal solution can be uniquely determined by applying the first order condition with respect to $P$ and $B$.

$$
\left\{\begin{array}{l}
\frac{\partial\left(R_{E U}(P, B) \cdot R_{C S P}(P, B)\right)}{\partial P}=0 \\
\frac{\partial\left(R_{E U}(P, B) \cdot R_{C S P}(P, B)\right)}{\partial B}=0
\end{array}\right.
$$

Rearranging Eq. 9, we get

$$
\frac{\partial R_{E U}}{\partial B} / \frac{\partial R_{E U}}{\partial P}=\frac{\partial R_{C S P}}{\partial B} / \frac{\partial R_{C S P}}{\partial P}
$$

Notice that $\frac{\partial R_{E U}}{\partial B} / \frac{\partial R_{E U}}{\partial P}$ is the slope of EU's indifferent revenue curve and $\frac{\partial R_{C S P}}{\partial B} / \frac{\partial R_{C S P}}{\partial P}$ is the slope of CSP's indifferent revenue curve. Obviously, the solution of Eq. 10 is Pareto Efficiency, reflected in Figure.1(b) is that the two curves $R_{\hat{C S P}}$ and $\hat{R_{E U}}$ are tangent to each other at point $G$, and neither of theirs can be further improved. By substituting Eq. 1 and Eq. 2, Eq. 10 can be further reduced to $U^{\prime}\left(B^{*}\right)=C_{C S P}^{\prime}\left(B^{*}\right)$. Since the equilibrium profile $\left(P^{*}, B^{*}\right)$ should also satisfy the EU's self-interest, which implies $P^{*}=U^{\prime}\left(B^{*}\right)$. Therefore, the optimal equilibrium strategy profile is $P^{*}=U^{\prime}\left(B^{*}\right)=C_{C S P}^{\prime}\left(B^{*}\right)$.

Theorem 2 shows that the optimal cloud storage contract could improve both parties' welfare and achieve Pareto efficiency by applying Nash Bargain Solution, where the marginal utility of EU equals to the marginal cost of CSP. This bargain process is possibly happen, especially under a competitive market, where EU will obtain a certain bargain power and CSP can not get high revenue by setting an arbitrary price. Such contract applied to the cloud services could better utilize internet resources. 


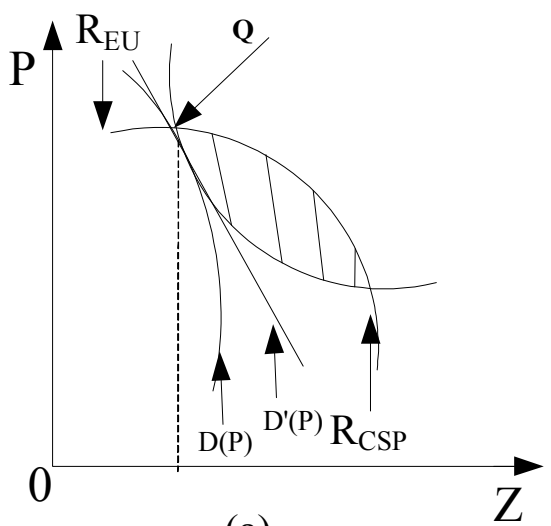

(a)

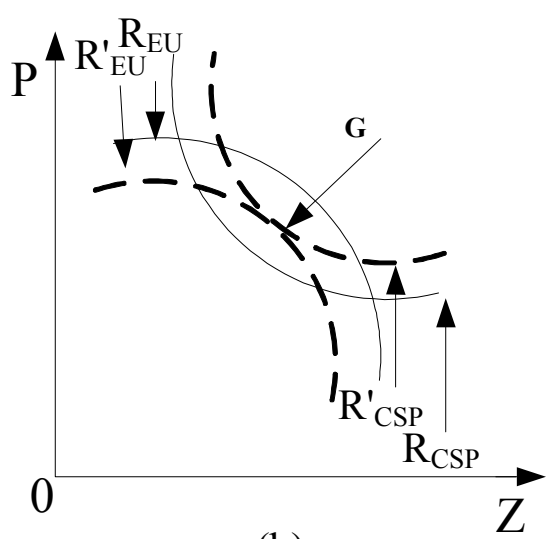

(b)

Fig. 1: (a) Noncooperative Equilibrium; (b) Pareto Improvement.

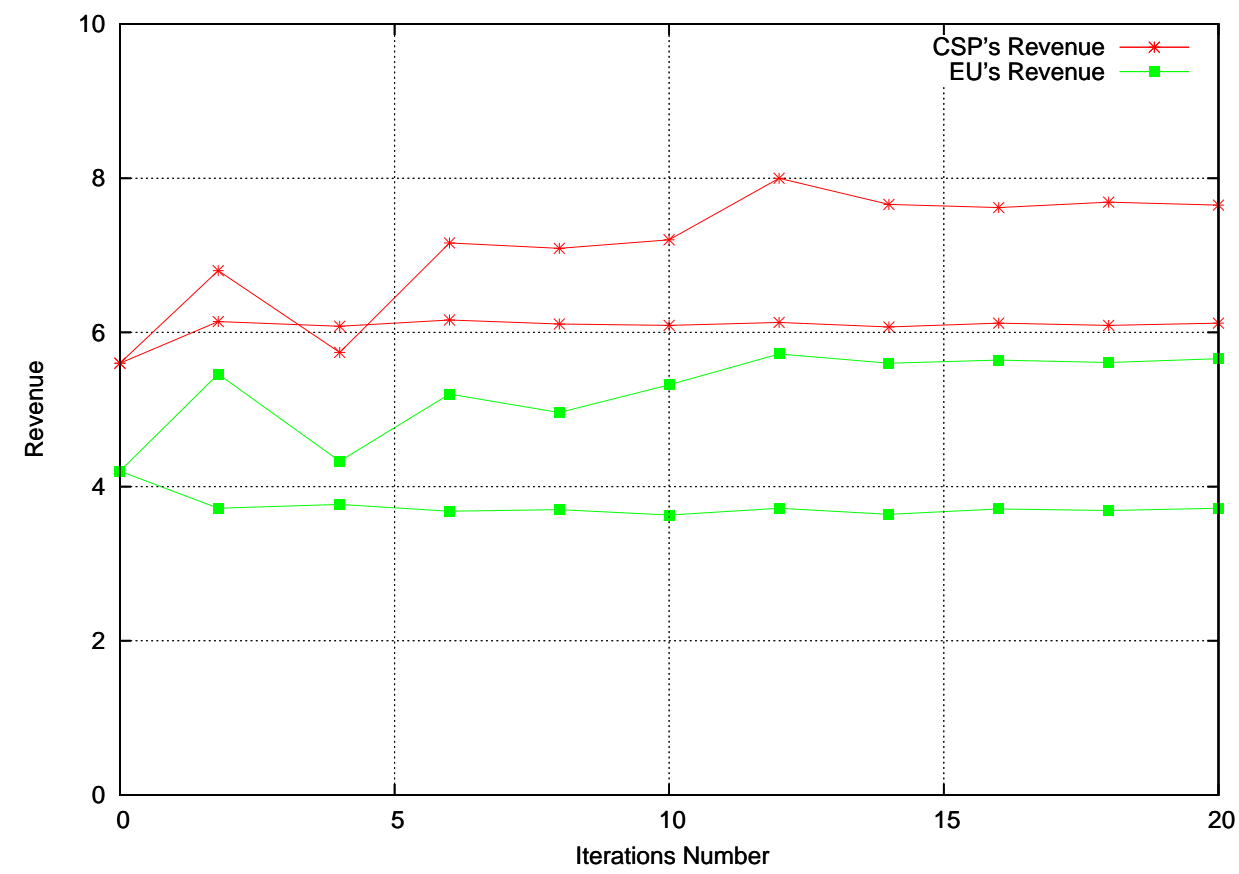

Fig. 2: Non-cooperative Revenue of Both Parties

\section{Numerical Analysis}

In this section, we provide numerical examples to illustrate the insight obtained from previous theoretical analysis. In particular, we focus on analyzing the changing revenue of the EU and CSP in cloud storage contract. Two scenarios are considered, i.e., non-cooperative game and bargain game.

We first investigate the cloud storage contract in non-cooperative game. Figure. 2 shows the evolution revenues of EU and CSP with a noncooperative equilibrium. We observe that the equilibrium number of iterations of noncooperative game is about 2, which is less than that in bargain equilibrium. The reason is that EU just needs to adjust its storage according to the given price of CSP.

Figure. 3 shows the long term gain of the two parties when the EU and CSP apply the Nash bargain process in cloud storage contract. Comparing with the result indicated in Figure.2, it can be seen that it takes more time for CSP and EU to make a revenue agreement in a bargain game, which is about 12 . In addition, both parties' revenue at the state of bargain equilibrium are 


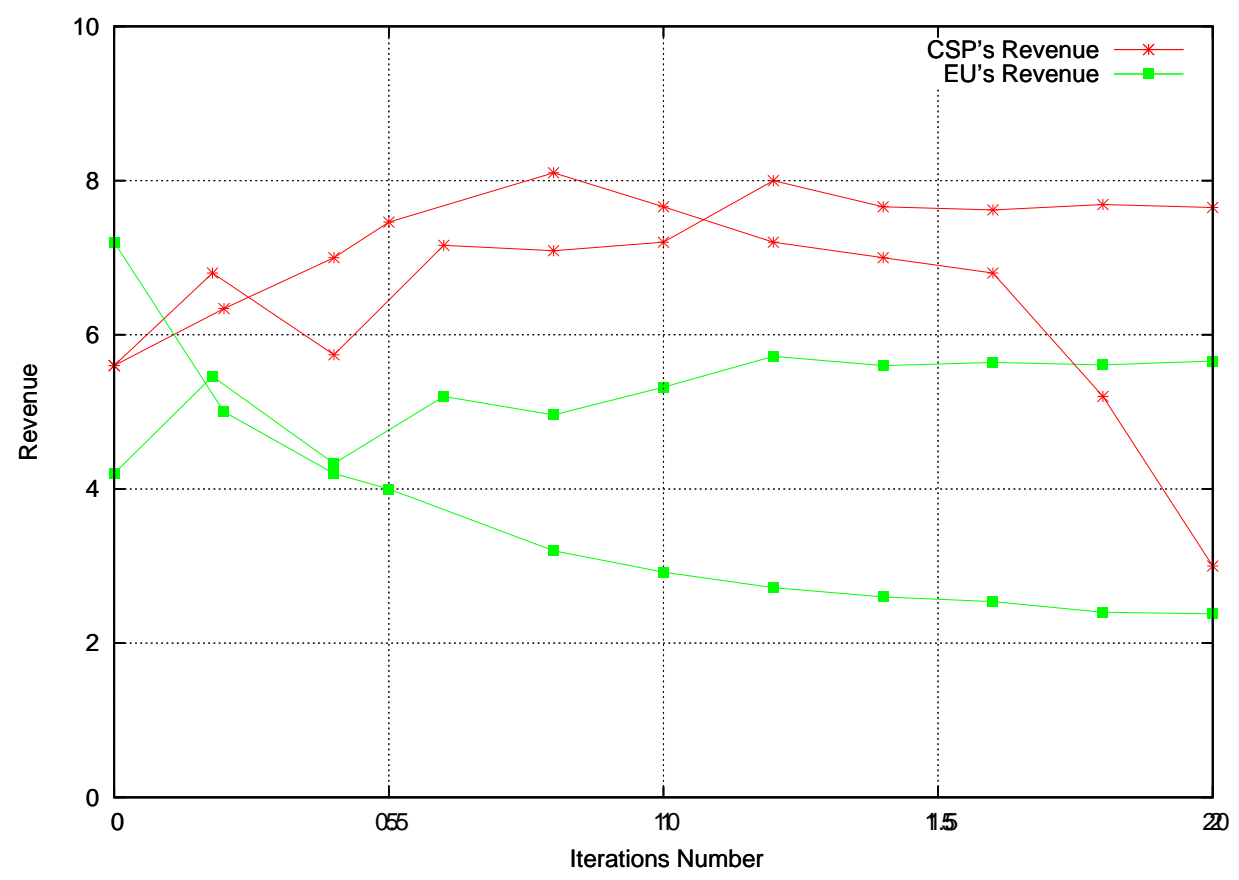

Fig. 3: Non-cooperative Revenue of Both Parties

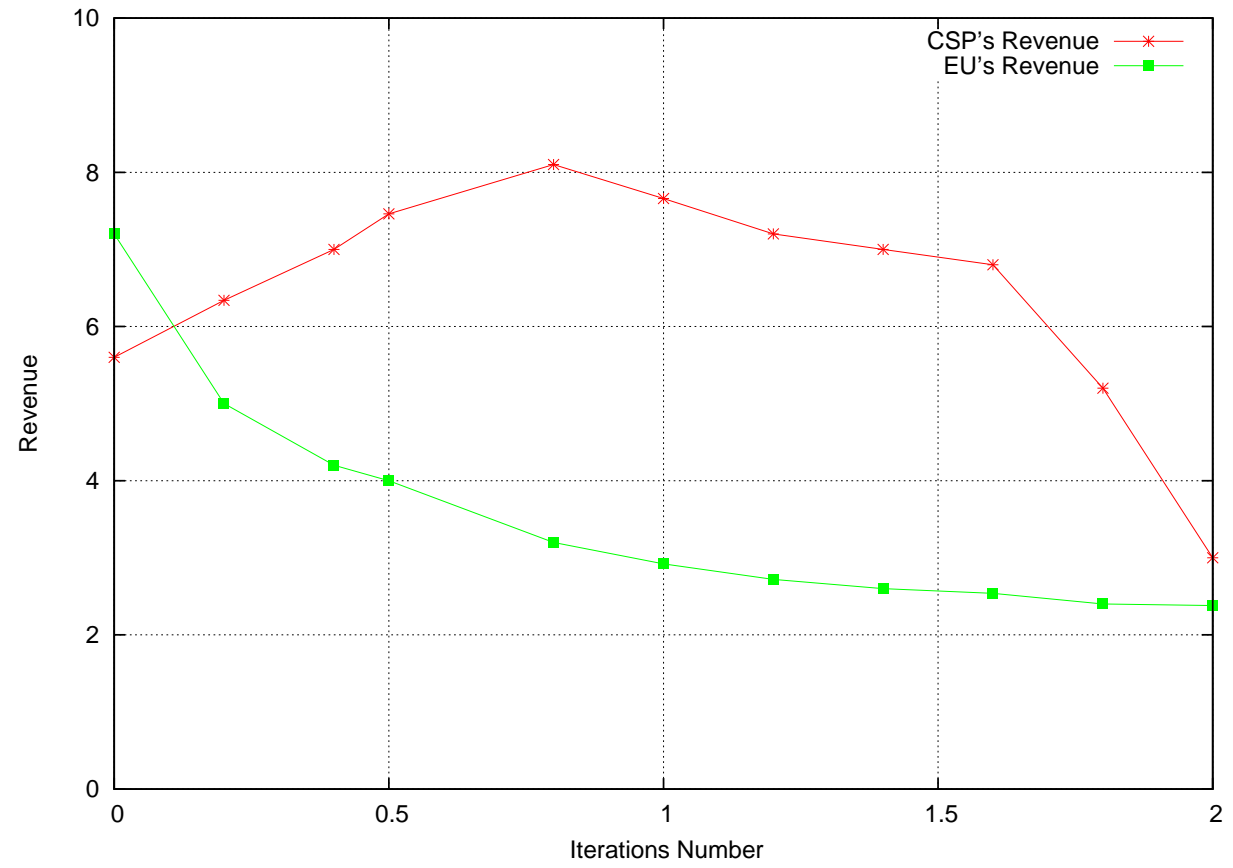

Fig. 4: Price $P$ vs Two Parties' Revenue 
improved compared to that in the noncooperative equilibrium, which is consistent with our previous analysis.

Lastly, we study the effect of the price of CSP on two parties' revenues. For the revenue curves shown in Figure.4, we can see that as price $p$ increases at the initial stage, the revenue of CSP increases. We can also see that there exists a maximal revenue of CSP when $p=0.8$, and then CSP's revenue decreases when $p$ continues increasing. These observations suggest that CSP can not improve its revenue by increasing price when the price achieves a certain level. Conversely, EU's revenue decreases monotonically with the increasing price. We notice that the larger $p$ results in very slow revenue decline of EU. This is because that, the higher $p$ is, the less cloud storage resource the EU will purchase.

\section{Conclusion}

In this paper, we model the parties involved in the cloud storage service as real economic entities, which are organized as a cloud storage contract. We formalize the interactions between EU and CSP as Stackelberg game and analyze the equilibrium strategies of the two parties. We show that the welfare of two parties involved in the cloud storage contract can be further enhanced to achieve the system optimality by applying Nash Bargain Solution(NBS). Our theoretical analysis and empirical studies both show that the bargain game can result in a win-win outcome. We hope that our analysis could shed lights on the deployment and evolution of cloud service.

\section{Acknowledgements}

This work is supported in part by a Fundamental Research Funds for the Central Universities (No.Z10921108) and in part by a National Nature Science Foundation of China (NSFC No. 61303226)

\section{References}

[1] M. Armbrust, A. Fox, R. Griffith, A.D. Joseph, R. Katz, and A. Konwinski. A view of cloud computing. In Commun. ACM, 2009.

[2] Potter L IversonMA, Ozguner F. Task allocation in a multiserver system. J Sched, pages 423 - 436, 2003.

[3] Christodoulou G, Koutsoupias E, and Kovacs A. Mechanism design for fractional scheduling on unrelated machines. In ICALP, 2007.

[4] J. Park and M. Schaar. Pricing and Incentives in Peer-toPeer Networks. In Proc. of IEEE Infocom, 2010.

[5] Amazon Simple Storage Service. http://aws.amazon.com/s3. 2008.

[6] Eucalyptus. http://eucalyptus.cs.ucsb.edu/. 2008.
[7] Righter R Koole G. Resource allocation in grid computing. J Sched, pages 163 - 173, 2008.

[8] C. Courcoubetis and R. Weber. Incentives for Large Peerto-Peer Systems. IEEE JSAC, 24(5):1034-1050, 2006.

[9] An B, Vasilakos AV, and Lesser V. Evolutionary stable resource pricing strategies. In ACM SIGCOMM, 2009.

[10] Andelman N, Azar Y, and Sorani M. Truthful approximation mechanisms for scheduling selfish related machines. STOC, page 69 C 82, 2005.

[11] Briest P and Krysta P. Approximation techniques for utilitarian mechanism design. STOC, pages 39-48, 2005.

[12] A. Mas-Colell, M. D. Whinston, and J. R. Green. Microeconomic Theory. In Oxford University Press, 1995.

[13] P. Dubey. Inefficiency of Nash Equilibria. Mathematics of Operations Research, 1986.

[14] K. Binmore, A. Rubinstein, and Asher Wolinsky. The Nash Bargaining Solution in Economic Modelling. In RAND Journal of Economics, 1986.

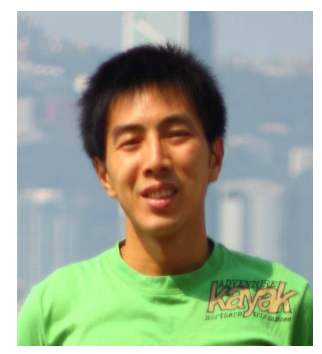

Libin Yang is an assistant professor in College of Information Engineering, Northwest A\& F University. He was a research associate in department of computing, the Hong Kong Polytechnic University, Hong Kong, from June 2009 to June 2011. He received the PHD degree from Northwestern Polytechnical University, China, in 2009. His current research interests include network economic, computer network and game theory.

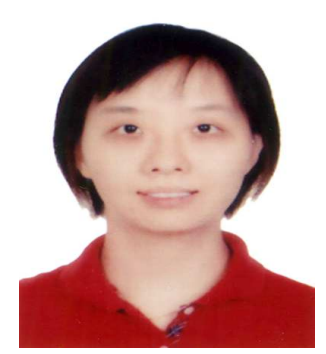

Xiaoyan Cai is an assistant professor in College of Information Engineering,Northwest A\& F University.She was a research associate in department of computing, the Hong Kong Polytechnic University,Hong Kong, from June 2009 to June 2011. She received the PHD degree from Northwestern Polytechnical University,China, in 2009. Her current research interests include game theory,information retrieval and machine learning. 